\title{
BMJ Open Multimorbidity in primary care in Portugal (MM-PT): a cross-sectional three-phase observational study protocol
}

\author{
Filipe Prazeres, ${ }^{1,2}$ Luiz Santiago ${ }^{1,3}$
}

To cite: Prazeres $\mathrm{F}$, Santiago L. Multimorbidity in primary care in Portugal (MMPT): a cross-sectional threephase observational study protocol. BMJ Open 2014;4: e004113. doi:10.1136/ bmjopen-2013-004113

- Prepublication history and additional material for this paper is available online. To view these files please visit the journal online (http://dx.doi.org/10.1136/ bmjopen-2013-004113).

Received 24 September 2013 Revised 21 January 2014 Accepted 24 January 2014

\section{CrossMark}

${ }^{1}$ Faculdade de Ciências da Saúde, Universidade da Beira Interior, Covilhã, Portugal ${ }^{2}$ Centro de Saúde de Aveiro, Aveiro, Portugal ${ }^{3}$ USF Topázio, Coimbra, Portugal

Correspondence to Dr Filipe Prazeres; filipeprazeresmd@gmail.com

\section{ABSTRACT}

Introduction: Multimorbidity is defined as the cooccurrence of more than one chronic disease in one person without assigning an index disease. This rapidly increasing phenomenon markedly influences patients' overall health, has major implications for effective provision of healthcare services and has a significant economic toll on individuals and society. Since Portugal is a country with a growing ageing population, a better understanding of the role of multimorbidity should be assessed. The aim of this study is to further the knowledge of the epidemiological factors associated with multimorbidity in Portugal, chiefly its prevalence and the health and social implications.

\section{Methods and analysis: This study protocol} describes a primary care nationwide three-phase study. The first phase is drawn to access the prevalence and patterns of multimorbidity. In the second phase, individual parameters are assessed, such as patients' health-related quality of life, perceived family support and unmet health needs of patients with multimorbidity. The third and last phase of this study aims to characterise general practitioners' knowledge, awareness and practices related to multimorbidity management.

Ethics and dissemination: The study will be conducted in accordance with the principles expressed in the Declaration of Helsinki. It has full approval from the Ethics Committee of the Faculty of Health Sciences, University of Beira Interior, and the Ethics Committee of the Central Health Region of Portugal. Study results will be published in peer-reviewed journals and presented at national and international conferences.

\section{INTRODUCTION}

Multimorbidity is defined as the co-occurrence of more than one chronic disease within one person without assigning an index disease. ${ }^{12}$ It is known that prevalence of multimorbidity increases with age, ${ }^{3}$ reaching over 90\%. ${ }^{4}$ Nevertheless, variations in prevalence of more than $60 \%$ have been reported between studies. These estimated differences may be due to sampling bias, period of collection and data source and also due to the broad definition of chronic diseases. ${ }^{5}$

Multimorbidity has been previously described by Martin Fortin as the rule and not the exception in primary care settings, ${ }^{3}$ where general practitioners (GPs) manage not only the acute but mostly the chronic conditions of their patients, ${ }^{6}$ which are often multiple. $^{7}$

Taking into account only disease-specific recommendations can lead to a considerable treatment burden for patients with multimorbidity. ${ }^{8}$ This in turn raises several other issues such as low compliance, ${ }^{9}$ treatment ineffectiveness ${ }^{10}$ and high treatment costs. ${ }^{11}$

Multimorbidity is also associated with lower life expectancy, ${ }^{12}$ higher hospital admissions ${ }^{13}$ and longer hospital stays. ${ }^{14}$ Quality of life, ${ }^{15}$ physical functioning ${ }^{16}$ and psychological health $^{17}$ are also negatively affected by multimorbidity.

Chronic diseases represent a significant cause of mortality and morbidity worldwide $\mathrm{e}^{18}$ and their increasing prevalence ${ }^{19}$ has a significant impact on patients' health and use of healthcare services.

Multimorbidity is, therefore, a subject which is rapidly becoming of great importance in primary care settings, with a pressing need for a better understanding of this phenomenon from multiple perspectives. This work aims to further the knowledge of the epidemiology of multimorbidity, regarding its prevalence and morbidity. Importantly, this will be the first study raising awareness of multimorbid conditions in adult population attending general practice in Portugal.

\section{Terminology}

For the purpose of defining multimorbidity, we will use the list of chronic conditions ${ }^{20} \mathrm{com}-$ piled by the Family Medicine Research Centre (FMRC), University of Sydney, available online at http://sydney.edu.au/medicine/fmrc/ classifications/DefiningChronicConditions.pdf. 
This list represents 147 ICPC-2 (International Classification of Primary Care) rubrics.

\section{Study objectives}

The primary objective of the study described in the protocol is to determine the prevalence of multimorbidity, quality of life, family support and unmet health needs of adult patients with multimorbidity attending primary care in Portugal.

Specific objectives are to:

- Describe the sociodemographic and clinical profiles of patients with multimorbidity in Portugal;

- Characterise the unmet health needs of Portuguese patients with multimorbidity;

- Evaluate the impact of multimorbidity on patients' health-related quality of life;

- Assess family support to patients with multimorbidity in Portugal;

- Analyse the relationship between multimorbidity, patients' health-related quality of life and family support;

- Describe Portuguese GPs' knowledge, awareness and practices related to the management of multimorbidity.

\section{METHODS AND ANALYSIS}

\section{Study design}

The study consists of three phases:

1. Cross-sectional, analytical study of the prevalence and patterns of multimorbidity in the adult population attending primary care in Portugal.

2. Cross-sectional, analytical study of patients' health-related quality of life, self-perceived family support and unmet health needs of adult patients with multimorbidity attending primary care in Portugal.

3. Descriptive before-after study of Portuguese GPs' knowledge, awareness and practices regarding multimorbidity and its management.

\section{Phase I: prevalence of multimorbidity in the adult population attending primary care in Portugal \\ Design}

Cross-sectional, analytical study.

\section{Setting}

Primary Care Centres in mainland Portugal will be randomly selected from the five Portuguese Healthcare Administrative Regions in order to obtain a national geographical representative sample.

\section{Sample size}

Since the prevalence of multimorbidity varies greatly across research literature and there is no available estimate for Portugal, a prevalence of $50 \%$ was assumed in order to maximise the sample size. For the study to be able to estimate a $95 \%$ CI for the prevalence of multimorbidity with a maximum precision error of $2.5 \%$, a total of 1500 patients should be recruited (Epidat 4.0-
Organización Panamericana de la Salud, Washington, DC, USA).

\section{Study procedures}

This phase of the study is expected to start in October 2013.

A two-step selection approach, based on the work of Fortin $e t a l l^{3}$ will be followed. First, GPs will be contacted and those who accept to participate will recruit their own patients. Assuming that a GP will be able to include at least 10 patients in a 3-week period, a total of 150 GPs has to be enrolled in the study. Considering a 30\% invitation response rate, a total of 500 GPs should be invited to participate in the study: 182 in North (36\%), 117 in Centre (23\%), 139 in Lisbon-Tejo Valley (28\%), 38 in Alentejo (8\%) and 22 in Algarve (4\%) in accordance with the distribution of the Portuguese adult population (18+ years) across the five mainland Portuguese Healthcare Administrative Regions.

The GPs to be invited will be randomly selected from an alphabetically ordered list prearranged per Healthcare Administrative Regions, using a random number generator (Independent Random Sampling). ${ }^{21}$

Enrolled GPs will be instructed to invite all adult ( $\geq 18$ years ) patients attending to the primary care consultation to participate in the study during 3 days on three consecutive weeks (Tuesday on week 1; Wednesday on week 2; and Thursday on week 3). Participants willing to participate in the study must give written informed consent and present willingness and ability to comply with the study requirements. Participants will be excluded if they are acutely unwell or refuse to participate.

\section{Data collection}

GPs will be responsible for collecting all data about each patient during their consultations and through the completion of a paper questionnaire developed specifically for this study. The patient's morbidities will be captured by GPs, using their knowledge of patient's history, patient's self-report and electronic and/or paper medical records of the patient. Morbidities will be collected using the ICPC-2 codes or the International Classification of Diseases-10th version (ICD-10), which are currently being used in Portuguese Primary Care Centres.

The personal information section of the Portuguese version of the EASY-Care questionnaire ${ }^{22}$ will be used to collect patients' social and demographic characteristics, namely gender, age, residence area, current marital status, number of years of formal education, living arrangements, professional status and self-perceived economic status through the question 'In general how do your family finances work out at the end of the month?'.

Data will be electronically stored in a database specifically designed for this study using MS Access 2010. Data will be encrypted and password protected. Information 
will be treated in strict confidentiality to protect the privacy of patients.

Paper copies of all informed consents will be retained in a locked file, separate from any study data.

\section{Statistical analysis}

A descriptive analysis will be performed to all study variables, namely the number of valid observations, mean, $\mathrm{SD}$, median and range for quantitative variables and absolute and relative frequencies for qualitative variables. Prevalence of multimorbidity (considering two definitions: $\geq 2$ or $\geq 3$ diseases) will be calculated together with corresponding 95\% CI. Moreover, the prevalence of multimorbidity will be estimated by subgroups, namely gender, age, residence area, current marital status, the number of years of formal education, living arrangements, professional status and selfperceived economic status. Univariate analysis will be conducted to study the associations between those characteristics and multimorbidity using $\chi^{2}$ test (qualitative characteristics) or t test/Mann-Whitney (quantitative characteristics). Multiple logistic regressions will be carried out considering the presence of multimorbidity as the dependent variable and patients' characteristics as the independent variables in order to calculate ORs and corresponding $95 \%$ CIs. Total number of morbidities by patient will also be summarised together with $95 \%$ CI, and multiple regressions may be performed to analyse its association with patients' characteristics. All analyses will be performed for both aforementioned definitions of multimorbidity. All tests will be two-sided using a significance level of 0.05 . Statistical analysis will be conducted using SPSS V.18.0 or higher.

Phase II: patients' health-related quality of life, perceived family support and unmet health needs of adult patients with multimorbidity attending primary care in Portugal Design

Cross-sectional, analytical study.

\section{Setting}

Primary Care Centres in the Centre region of Portugal will be randomly selected within each Care Centres Grouping (ACES and ULS) in order to obtain a regional geographical representative sample.

\section{Sample size}

A total of 500 patients should be included in phase II in order to obtain $95 \%$ CIs for proportions with a maximum precision error of $4.5 \%$ and $95 \%$ CIs for SF-12 mean scores with a precision error of 4.5 (assuming an SD of 50).

\section{Study procedures}

Phase II of the study is expected to start in January 2014.

A two-step approach will be considered as in phase I. Assuming that each GP will enrol 10 patients and that $30 \%$ of the invited GPs will accept to participate, then about 170 GPs within the randomly selected Care Centres should be invited to participate in this phase of the study.

Primary Care Centres will be randomly selected (Independent Random Sampling) ${ }^{21}$ and all GPs within those centres will be invited to participate (until 170 GPs are recruited).

By purposive sampling, enrolled GPs will select adult ( $\geq 18$ years) patients with multimorbidity and with at least one of four morbidities: hypertension, diabetes, asthma and/or osteoarthritis. These morbidities were selected based on the Portuguese Directorate-General of Health (DGS-Direção-Geral da Saúde) efforts to implement specific national recommendations for the diagnosis, treatment and control of these conditions, which have high prevalence and are associated with low quality of life. ${ }^{23-26}$

The investigator or a previously trained research associate (GP/GP trainee) will interview the patients at their convenience, either in the GPs office or at their home. Participants willing to participate in the study must give written informed consent and present willingness and ability to comply with the study requirements. Participants will be excluded if they are acutely unwell or refuse to participate.

\section{Data collection}

All data will be collected using a paper questionnaire. Patients' morbidities, social and demographic characteristics will be registered using the same methodology as described in phase I. Health-related quality of life will be evaluated using the Portuguese Short Form-12 Health Status Questionnaire (SF-12), ${ }^{27}$ derived from the Medical Outcomes Study 36-Item Short Form Health Survey (SF-36), ${ }^{28}$ through interview of the patients. The Portuguese Family APGAR (Adaptation, Partnership, Growth, Affection and Resolve) Questionnaire ${ }^{29} 30$ that evaluates family function in five dimensions ${ }^{31}$ will be used to measure the perceived family support of patients with multimorbidity.

On the basis of the literature, ${ }^{32-36}$ a patients' unmet health needs questionnaire was drafted. The unmet need for the following services will be evaluated: medical, surgical and dental care, prescription medications, mental healthcare or counselling, and eyeglasses or other technical aid. These will be evaluated by a set of general questions, such as 'During the past 12 months, was there a time when you wanted/needed medical care but couldn't get it at that time?' If an unmet health need is stated, available reasons to explain it are: Couldn't you get off work? Were you too sick? Didn't you have a way to get there? Did you have responsibilities to take care of someone? Were you afraid to leave home because of personal safety? Did you have other more important things to take care of? Did you have to spend your money for food, clothing, housing, etc? These questions will be translated to Portuguese by the study investigator and the translation will be subsequently reviewed by an 
independent GP expert. Any disagreements will be discussed between both in order to achieve a consensus. Furthermore, a pretest will be undertaken and the translated questions will be applied to 50 participants prior to patients enrolment phase in order to verify participants' comprehensibility of those questions.

\section{Statistical analysis}

Descriptive statistics will be computed for all variables together with $95 \%$ CIs whenever relevant and applicable. Associations between qualitative-independent variables will be tested using $\chi^{2}$ test. Comparisons between two or more independent groups regarding a quantitative variable are to be conducted using analysis of variance (ANOVA) or Kruskal-Wallis non-parametric test, if normality assumption is not met. ANCOVA may also be used to adjust for potential confounding factors. Associations between quantitative independent variables will be analysed using Pearson's or Spearman's correlation coefficient depending on normality assumption. All tests will be two-sided, considering a significance level of 0.05 .

\section{Phase III: Portuguese GPs' knowledge, awareness and practices regarding multimorbidity and its management Design}

Before-after, descriptive study.

Since there is a lack of research on multimorbidity in Portugal, we believe that this concept is not usually taken into account in daily practice. We also have confidence in that the dissemination of the results of parts I and II of the study will have the capability of raising awareness and change behaviour towards patients with multimorbidity. To test these hypotheses, a before-after study was designed.

\section{Setting}

Selected Primary Care Centres in two districts of the Centre region of Portugal (Coimbra and Aveiro).

\section{Sample size}

Since this is a descriptive study, no formal calculations were performed to estimate the sample size. Notwithstanding, we are willing to include at least $10 \%$ of the population of GPs in these two districts corresponding to a sample size of approximately $60 \mathrm{GPs}$.

\section{Study procedures}

GPs working in Primary Care Centres in the districts of Coimbra and Aveiro will be recruited by chain-referral sampling.

\section{Data collection}

Prior to the start of phase I of the study, an online questionnaire with open text format questions will be used to evaluate GPs' knowledge (definition of multimorbidity), awareness (relevance of multimorbidity in daily practice) and practices (management of multimorbidity). This will be followed by a second application of the same questionnaire after the distribution of a flyer with the results of phases I and II of the study.

This questionnaire will have a semantic validation by two distinct groups. The first is composed of experts in multimorbidity and the second by possible respondents, in our case, GPs.

\section{Statistical analysis}

Before-after collected answers will be listed and analysed by the study investigator. If possible, answers will be converted into qualitative variables by the study investigator in order to evaluate knowledge, awareness and practices. This process will also be performed by an independent expert and results will be crosschecked. Discrepancies will be further discussed until a consensus is achieved. Results obtained will be summarised using descriptive statistics as previously described.

Contributors FP and LS were involved in designing of the study. FP was involved in writing of the manuscript. All authors read and approved the final manuscript draft.

Funding This research received no specific grant from any funding agency in the public, commercial or not-for-profit sectors.

\section{Competing interests None.}

Ethics approval The study will be conducted in accordance with the principles expressed in the Declaration of Helsinki. It has full approval from the Ethics Committee of the Faculty of Health Sciences, University of Beira Interior and the Ethics Committee of the Central Health Region of Portugal. Study results will be published in peer-reviewed journals and presented at national and international conferences.

Provenance and peer review Not commissioned; externally peer reviewed.

Open Access This is an Open Access article distributed in accordance with the Creative Commons Attribution Non Commercial (CC BY-NC 3.0) license, which permits others to distribute, remix, adapt, build upon this work noncommercially, and license their derivative works on different terms, provided the original work is properly cited and the use is non-commercial. See: http:// creativecommons.org/licenses/by-nc/3.0/

\section{REFERENCES}

1. van den Akker M, Buntinx F, Knottnerus JA. Comorbidity or multimorbidity. Eur J Gen Pract 1996;2:65-70.

2. van den Akker M, Buntinx F, Roos S, et al. Problems in determining occurrence rates of multimorbidity. J Clin Epidemiol 2001;54:675-79.

3. Fortin M, Bravo G, Hudon C, et al. Prevalence of multimorbidity among adults seen in family practice. Ann Fam Med 2005;3:223-8.

4. Fortin M, Stewart M, Poitras ME, et al. A systematic review of prevalence studies on multimorbidity: toward a more uniform methodology. Ann Fam Med 2012;10:142-51.

5. Stewart M, Fortin M, Britt HC, et al. Comparisons of multi-morbidity in family practice-issues and biases. Fam Pract 2013;30:473-80.

6. Europe W. The European definition of general practice/family medicine. http://www.woncaeurope.org/sites/default/files/documents/ Definition\%203rd\%20ed\%202011\%20with\%20revised\%20wonca\% 20tree.pdf. Published 2002. (accessed 24 September 2013).

7. Flocke SA, Frank SH, Wenger DA. Addressing multiple problems in the family practice office visit. J Fam Pract 2001;50:211-16.

8. Hughes LD, McMurdo ME, Guthrie B. Guidelines for people not for diseases: the challenges of applying UK clinical guidelines to people with multimorbidity. Age Ageing 2013;42:62-9.

9. Townsend A, Hunt K, Wyke S. Managing multiple morbidity in mid-life: a qualitative study of attitudes to drug use. BMJ 2003;327:837-40.

10. Fortin M, Soubhi $\mathrm{H}$, Hudon $\mathrm{C}$, et al. Multimorbidity's many challenges. BMJ 2007;334:1016-17. 
11. Wolff JL, Starfield B, Anderson G. Prevalence, expenditures, and complications of multiple chronic conditions in the elderly. Arch Intern Med 2002;162:2269-76.

12. Gijsen R, Hoeymans N, Schellevis FG, et al. Causes and consequences of comorbidity: a review. J Clin Epidemiol 2001;54:661-74.

13. Freund T, Kunz CU, Ose D, et al. Patterns of multimorbidity in primary care patients at high risk of future hospitalization. Popul Health Manag 2012;15:119-24.

14. Rochon PA, Katz JN, Morrow LA, et al. Comorbid illness is associated with survival and length of hospital stay in patients with chronic disability. A prospective comparison of three comorbidity indices. Med Care 1996;34:1093-101.

15. Fortin M, Bravo G, Hudon C, et al. Relationship between multimorbidity and health-related quality of life of patients in primary care. Qual Life Res 2006;15:83-91.

16. Kadam UT, Croft PR, North Staffordshire GPCG. Clinical multimorbidity and physical function in older adults: a record and health status linkage study in general practice. Fam Pract 2007;24:412-19.

17. Fortin M, Bravo G, Hudon C, et al. Psychological distress and multimorbidity in primary care. Ann Fam Med 2006;4:417-22.

18. Busse R, Bluemel M, Scheller-Kreinsen D, et al. Tackling Chronic Disease in Europe. Strategies, Interventions and Challenges. http:// pns.dgs.pt/files/2010/05/Doen\%C3\%A7as-cr\%C3\%B3nicas_EU Maio_2010-2.pdf Published 2010. (accessed 24 Sep 2013).

19. Uijen AA, van de Lisdonk EH. Multimorbidity in primary care: prevalence and trend over the last 20 years. Eur J Gen Pract 2008;14(Suppl 1):28-32.

20. O'Halloran J, Miller GC, Britt H. Defining chronic conditions for primary care with ICPC-2. Fam Pract 2004;21:381-6.

21. Fox N, Hunn A, Mathers N. Sampling and sample size calculation. The NIHR RDS for the East Midlands/Yorkshire \& the Humber, 2007.
22. Sousa L, Figueiredo D. (In)dependência na população idosa: um estudo exploratório na população portuguesa. Psychol 2003;33:109-22.

23. DGS. Diagnóstico, Tratamento e Controlo da Hipertensão Arterial. Lisboa: DGS, 2004.

24. DGS. National Programme for the Prevention and Control of Diabetes. Lisbon: DGS, 2008.

25. DGS. National Programme for Asthma Control. Lisbon: DGS, 2005

26. DGS. National Programme against Rheumatic Diseases. Lisbon: DGS, 2005.

27. Pais-Ribeiro J. O importante é a saúde: estudo de adaptação de um instrumento para avaliar o estado de saúde. Lisboa: Fundação Merck Sharp \& Dohme, 2005.

28. Ware J Jr, Kosinski M, Keller SD. A 12-Item Short-Form Health Survey: construction of scales and preliminary tests of reliability and validity. Med Care 1996;34:220-33.

29. Imperatori E. Ficha familiar dos centros de saúde: vantagens e dificuldades. Rev Port Sau Pub 1985;3:51-6.

30. Agostinho M, Rebelo L. Família: do conceito aos meios de avaliação. Rev Port Clin Geral 1988;32:6-17.

31. Smilkstein G. The family APGAR: a proposal for a family function test and its use by physicians. J Fam Pract 1978;6:1231-9.

32. Baggett TP, O'Connell JJ, Singer DE, et al. The unmet health care needs of homeless adults: a national study. Am J Public Health 2010;100:1326-33.

33. Shi L, Stevens GD. Vulnerability and unmet health care needs. The influence of multiple risk factors. J Gen Intern Med 2005;20:148-54

34. Diamant AL, Hays RD, Morales LS, et al. Delays and unmet need for health care among adult primary care patients in a restructured urban public health system. Am J Public Health 2004;94:783-9.

35. Newacheck PW, Hughes DC, Hung YY, et al. The unmet health needs of America's children. Pediatrics 2000;105(4 Pt 2):989-97.

36. Ayanian JZ, Weissman JS, Schneider EC, et al. Unmet health needs of uninsured adults in the United States. JAMA 2000;284:2061-9. 